\title{
BRAIDS AND THE JONES POLYNOMIAL
}

\author{
JOHN FRANKS AND R. F. WILLIAMS
}

\begin{abstract}
An important new invariant of knots and links is the Jones polynomial, and the subsequent generalized Jones polynomial or two-variable polynomial. We prove inequalities relating the number of strands and the crossing number of a braid with the exponents of the variables in the generalized Jones polynomial which is associated to the link formed from the braid by connecting the bottom ends to the top ends. We also relate an exponent in the polynomial to the number of components of this link.
\end{abstract}

In [J] Vaughan Jones introduced a new polynomial invariant of oriented knots and links in 3-space. Subsequently a number of researchers (Freyd and Yetter, Hoste, Lickorish and Millett, and Ocneanu; see [FYHLMO]) independently realized that this could be generalized to produce an invariant which is a Laurent polynomial of two variables and which specializes to give both the invariant of $[\mathbf{J}]$ and the classical Alexander polynomial (see $[\mathbf{R}]$ ).

More precisely, there is a function $P$ from isotopy classes of oriented links to $Z\left[x, x^{-1}, y, y^{-1}\right]$. If $L$ is an oriented link we will write $P(L)=j(x, y)$. By abuse of notation we will also write $\mathcal{P}(b)$ for $b$ an oriented braid, meaning, of course, the invariant associated to the oriented link determined by closing up $b$ in the usual way.

Several equivalent ways of codifying this invariant are described in [FYHLMO]. We have chosen the approach of Lickorish and Millett. Hence our $\mathcal{P}(K)=j(x, y)$ is precisely their $P_{K}$ with $x$ and $y$ substituted for $l$ and $m$ respectively (see [FYHLMO] and [L-M]).

There appears as yet to be no consensus on a name for this two-variable invariant. In order to avoid awkward circumlocutions and being unable to amalgamate the names of the six or eight individuals who actually discovered it, we have chosen to refer to it as the generalized Jones polynomial, or Jones polynomial for short, when there is no likelihood of confusion with the original Jones polynomial.

This polynomial, which we will denote $j(x, y)$, is characterized by Figure 1 .

The interpretation of this is as follows: Given a regular projection of a link $K_{+}$ with a crossing pictured as below, one can form two new links $K_{-}$and $K_{0}$ which are identical to $K_{+}$except the one crossing is changed as shown. When this is done the Jones polynomials of the three links are related as in the formula. It is a remarkable fact that this together with the normalization that $P$ (unknot) $=1$, uniquely determines a Laurent polynomial $j(x, y)$ and that this polynomial is a link invariant, i.e., is independent of the projection. This result can be found in $[\mathbf{L}-\mathbf{M}]$. This formula, in fact, gives a good method for recursively computing $j(x, y)$. One

Received by the editors January 13, 1986.

1980 Mathematics Subject Classification (1985 Revision). Primary 57M25.

Research supported in part by NSF Grant MCS 8301067. 


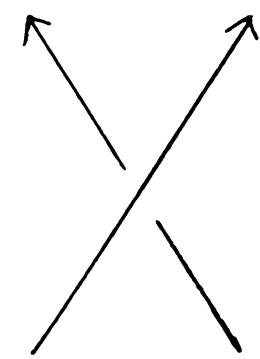

$K_{+}$

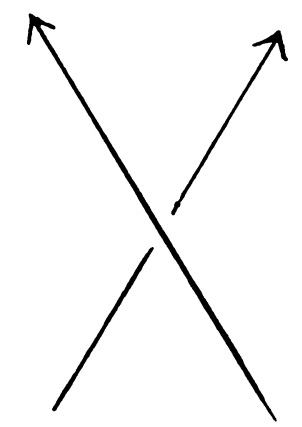

$K_{-}$

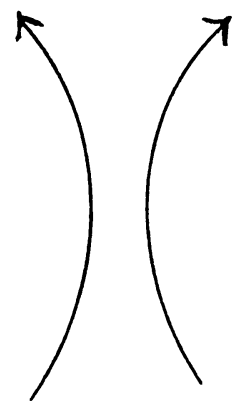

$K_{0}$

$$
\begin{gathered}
x P\left(K_{+}\right)+x^{-1} P\left(K_{-}\right)+y P\left(K_{0}\right)=0 . \\
\text { FIGURE } 1
\end{gathered}
$$

can start from scratch or use the fact that for a trivial link of $k$ components $j(x, y)$ equals $\mu^{k-1}$ where $\mu=-\left(x+x^{-1}\right) / y$.

A number of computer programs now exist for computing $j(x, y)$. We would like to thank Henry Cejtin, who wrote such a program for us. Without access to quick and easy computation of $j(x, y)$ for various braids our work would not have been possible.

In this article we focus on the application of the invariant $j(x, y)$ to the study of braids. For a general reference on braids and the braid group we refer the reader to $[\mathbf{B i r}]$.

We understand that many of our results and in particular the main result below have also been obtained independently by Hugh Morton $[\mathbf{M}]$.

This main result, which is (1.9) in the text, relates the number of strands in a braid, the crossing number of the braid and exponents in the associated Jones polynomial. The crossing number $c$ of an oriented braid is the number of positive crossings minus the number of negative crossings. We warn the reader that there appears to be no universal convention for what is positive and what is negative. For clarity we have chosen to adopt the convention used in $[\mathbf{J}]$ and $[\mathbf{L}-\mathbf{M}]$, though it is not our favorite, and we will call positive a crossing like that in the picture $K_{+}$above, and, of course, $K_{-}$is negative. The crossing number $c$ is also equal to the exponent sum when the braid is written as a word in the standard generators $\left\{\sigma_{i}\right\}, 1 \leq j \leq n-1$ (see [Bir]). Our main result (Corollary 1.9) is the following.

THEOREM. If $b$ is a braid on $n$ strands with (signed) crossing number $c$, then

$$
n-c-1 \geq e_{\max } \geq e_{\min } \geq 1-n-c
$$

where $e_{\max }$ is the largest exponent of $x$ in the Jones polynomial $j(x, y)$ associated to the link formed from the braid, and $e_{\min }$ is the smallest exponent.

The braid number $\beta$ of a link $L$ is the smallest number $n$ such that $L$ is combinatorially equivalent to a braid on $n$ strands. An easy corollary of the result above is given as (1.10) below. 
COROLlARY. If $L$ is a link with braid number $\beta$ and Jones polynomial $j(x, y)$ then

$$
\beta \geq \frac{\left(e_{\max }-e_{\min }\right)}{2}+1 .
$$

Here $e_{\max }$ and $e_{\min }$ are respectively the largest and smallest exponents of $x$ in $j(x, y)$.

We also show in Proposition (1.11) that the smallest exponent of $y$ in $j(x, y)$ is $1-k$ where $k$ is the number of components of the link. (See Theorem 15 of $[\mathbf{J}]$ for another way of extracting this information.)

In $\S 2$ we investigate positive braids. For them it is more enlightening to codify the information in $j(x, y)$ differently. We show in Theorem (2.2) that for positive braids one can translate $j(x, y)$ into a polynomial $J(R, C, T)$ in three variables from which information about the braid is easily read off. Among the nice properties of $J$ are that it is a genuine polynomial (no negative exponents), all its coefficients are positive, and if $R, C, T$ are assigned degrees 1,2 , and -1 respectively, then it is homogeneous of degree $1-n+c$ where $n$ is the number of strands and $c$ is the exponent sum of the positive braid. One can also read off the number of components of the link for $J(R, C, T)$.

\section{Computation trees.}

(1.1) DEFINITION. A computation tree $\Gamma$ is a connected, rooted, oriented binary tree, with each node labeled by a braid word and each edge labeled by a monomial, which satisfies the following properties:

If $N$ is any nonterminal node and is labeled with braid word $b$, and $N_{0}, N_{1}$ are the two child nodes of $N$ and are labeled with $b_{0}$ and $b_{1}$ respectively, then $P(b)=p_{0} \mathcal{P}\left(b_{0}\right)+p_{1} \mathcal{P}(b)$ where $p_{0}$ and $p_{1}$ are the monomials labeling the edges from $N$ to $N_{0}$ and $N_{1}$ respectively.

Obviously a computation tree is so named because one can compute $j$ of the root braid word by multiplying $j$ of each terminal node by the product of all the monomials along the path from this terminal to the root and then summing over all terminal nodes.

We shall be interested in computation trees with additional properties which we now describe. We will denote by $(b, n)$ a braid $b$ on $n$ strands.

(1.2) DEFINITION. An invariant Markov move consists of

(1) Replacing $(b, n)$ by $\left(b^{\prime}, n\right)$ where $b$ is conjugate to $b^{\prime}$, or

(2) Replacing $\left(b \sigma_{n-1}, n\right)$ by $(b, n-1)$ if $b$ is a word in the generators $\sigma_{1}, \ldots, \sigma_{n-2}$.

(3) The reverse of (2).

The usual Markov move $\left(b \sigma_{n-1}^{-1}, n\right) \rightarrow(b, n-1)$ is specifically disallowed by our definition.

Notice that if $(b, n)$ is related to $\left(b^{\prime}, n^{\prime}\right)$ by a sequence of invariant Markov moves, then $n-c=n^{\prime}-c^{\prime}$ where $c$ is the number of positive (right-handed) crossings in $b$ minus the number of negative crossings in $b$ and $c^{\prime}$ is the corresponding number for $b^{\prime}$. This is an important invariant quantity which we shall use subsequently, and this explains our choice of terminology.

We shall also be interested in Conway splits on braids. By this we mean starting with a word $(b, n)$, where $b=\alpha \sigma_{i}^{e} \beta$ and $e= \pm 1$, and producing two new words 
$\left(b_{0}, n\right)$ and $\left(b^{-}, n\right)$ where $b_{0}=\alpha \beta$ and $b^{-}=\alpha \sigma_{i}^{-e} \beta$. In this framework our basic relation for the Jones polynomial says

$$
\mathcal{P}((b, n))=p_{0} \mathcal{P}\left(\left(b_{0}, n\right)\right)+p^{-\mathcal{P}}\left(\left(b^{-}, n\right)\right)
$$

where

$$
p_{0}= \begin{cases}-x y & \text { if } e=-1 \\ -x^{-1} y & \text { if } e=1\end{cases}
$$

and

$$
p^{-}= \begin{cases}-x^{2} & \text { if } e=-1 \\ -x^{-2} & \text { if } e=1\end{cases}
$$

(1.3) Definition. An invariant computation tree $\Gamma$ is a computation tree satisfying the additional properties:

(1) If $\gamma$ is a node labeled by $(b, n)$ and its children $\gamma_{0}, \gamma_{1}$ are labeled by $\left(b_{0}, n_{0}\right)$ and $\left(b_{1}, n_{1}\right)$, then $\left(b_{0}, n_{0}\right)$ and $\left(b_{1}, n_{1}\right)$ can be obtained by first performing a sequence of invariant Markov moves on $(b, n)$ and then doing a Conway split.

(2) Each terminal node is labeled with the empty braid word on some number of strands (which may be different for different terminal nodes).

Notice that in order to be a computation tree the edges from $\gamma$ to $\gamma_{0}$ and $\gamma_{1}$ must be labeled with the monomials $p_{0}$ and $p^{-}$described in the discussion of Conway splits. These monomials depend only on the exponent $e$ of the generator $\sigma_{i}$ which one is changing in the Conway move.

We have not yet shown that invariant computation trees exist, but before doing so we derive an important property they have.

(1.4) Definition. Let $\chi(b, n)=n-c$ where $c$ is the sum of the exponents of $b$; i.e., $c$ equals the number of positive crossings minus the number of negative crossings in $b$.

(1.5) LEMMA. If $\Gamma$ is an invariant computation tree and $\gamma$ is a nonterminal node labeled with $(b, n)$ and $\gamma^{\prime}$ is one of its children labeled with $\left(b^{\prime}, n^{\prime}\right)$ then

$$
\chi(b, n)=\chi\left(b^{\prime}, n^{\prime}\right)+e_{x}
$$

where $e_{x}$ is the exponent of $x$ in the monomial labeling the edge joining $\gamma$ to $\gamma^{\prime}$.

Proof. The braid word $\left(b^{\prime}, n^{\prime}\right)$ was obtained from $(b, n)$ by first performing invariant Markov moves on $(b, n)$ and then doing a Conway split. Invariant Markov moves do not change the quantity $\chi(b, n)$ (as we remarked immediately after their definition). A Conway split produces from $(b, n)$ two braids $\left(b_{0}, n\right)$ and $\left(b^{-}, n\right)$. For $\left(b_{0}, n\right)$ the exponent sum has been changed by \pm 1 and the exponent of $x$ in the monomial labeling the edge from $\gamma$ to $\gamma^{\prime}$ is $\mp 1$ (see the discussion of Conway splits above). Likewise, for $\left(b^{-}, n\right)$ the exponent sum $c$ has changed by \pm 2 and the exponent of $x$ in the monomial is $\mp 2$.

If $\Gamma$ is a computation tree with root $\gamma_{0}$ and $\gamma$ is another node we define the rootpath monomial of $\gamma$ to be the product of all monomials labeling the edges which join $\gamma$ to $\gamma_{0}$. We will denote it $P(\gamma)$. 
(1.6) LEMMA. Suppose $\Gamma$ is an invariant computation tree with root $\gamma_{0}$ labeled by $\left(b_{0}, n_{0}\right)$ and node $\gamma$ labeled by $(b, n)$, then

$$
\chi\left(b_{0}, n_{0}\right)=\chi(b, n)+\operatorname{deg}_{x} P(\gamma),
$$

where $\operatorname{deg}_{x}$ denotes the exponent of $x$ in the monomial $P(\gamma)$.

PROOF. This follows immediately from Lemma (1.5) by induction.

(1.7) THEOREM. For any braid $(b, n)$ there exists an invariant computation tree $\Gamma$ whose root is labeled by $(b, n)$.

PROOF. We prove the result by induction on the length of the word $b$. If $b$ is the empty word the result is trivial, so suppose $b$ has length $k$ and the result is true for words of length $<k$.

By a sequence of Conway splits we want to convert $b$ to a positive braid $(\widehat{b}, n)$ where $\widehat{b}$ is obtained from $b$ by changing all the exponents of generators in $b$ to be positive.

Each Conway move at a node produces two children of that node. One of the children will be labeled with $\left(b_{0}, n\right)$ where $b_{0}$ has length $k-1$ (see the description of Conway moves above). Hence, by the induction hypothesis the remainder of the tree emanating from this child can be completed. The other child will be labeled with $\left(b^{-}, n\right)$ where $b^{-}$has length $k$ but one of the exponents in $b$ has had its sign changed to make it more like $\widehat{b}$. After repeating this finitely many times we arrive at a partial tree which is complete except for a single dangling node $\widehat{\gamma}$ labeled by $(\widehat{b}, n)$.

So far we have used no Markov moves, only Conway splits. But by Proposition (2.1) below, the positive braid $(\widehat{b}, n)$ can be converted by invariant Markov moves to $\left(b^{\prime}, n^{\prime}\right)$ where either $b^{\prime}$ is empty or $b^{\prime}=\alpha \sigma_{i}^{2}$ for some $i$. If we now perform a Conway split on a crossing represented by one of the $\sigma_{i}$ 's the two children will be labeled by $\left(\alpha \sigma_{i}, n^{\prime}\right)$ and $\left(\alpha, n^{\prime}\right)$ (since $\left.\alpha \sigma_{i} \sigma_{i}^{-1}=\alpha\right)$. Both of these words have length less than $k$, so by the induction hypothesis the invariant computation tree can be completed.

(1.8) THEOREM. Let $(b, n)$ be a braid word with Jones polynomial $j(x, y)$ and exponent sum (or signed crossing number) $c$. Then

$$
j(x, y)=x^{n-c-1} Q\left(y, y^{-1}, 1+x^{-2}\right)
$$

where $Q$ is a polynomial (no negative exponents) in three variables.

PROOF. Let $\Gamma$ be an invariant computation tree whose root is labeled with $(b, n)$ and let $T$ be the set of terminal nodes of $\Gamma$. If $\gamma \in T$ it is labeled with $(\phi, r)$ and the Jones polynomial of $(\phi, r(\gamma))$ is $\mu^{r(\gamma)-1}$ where $\mu=-\left(x^{-1}+x\right) / y$, and $r(\gamma)$ is the number of strands (or number of components since $\phi$ is the empty word).

If $P(\gamma)$ is the root-path monomial for $\gamma$, then by (1.5)

$$
n-c=\chi(b, n)=\chi(\phi, r(\gamma))+\operatorname{deg}_{x} P(\gamma)=r(\gamma)+\operatorname{deg}_{x} P(\gamma) .
$$

Hence for some $s(\gamma)$,

so

$$
P(\gamma)= \pm y^{s(\gamma)} x^{n-c-r(\gamma)},
$$

$$
P(\gamma) \mu^{r(\gamma)-1}= \pm y^{p(\gamma)} x^{n-c-1}\left(1+x^{-2}\right)^{r(\gamma)-1}
$$

where $p(\gamma)=s(\gamma)-r(\gamma)+1$. 
Now the Jones polynomial of $(b, n)$ is given by

$$
j(x, y)=\sum_{\gamma \in T} P(\gamma) \mu^{r(\gamma)-1}
$$

since $\Gamma$ is a computation tree and the Jones polynomial of a terminal node $\gamma$ is $\mu^{r(\gamma)-1}$. Thus we conclude

$$
j(x, y)=\sum_{\gamma \in T} \pm y^{p(\gamma)} x^{n-c-1}\left(1+x^{-2}\right)^{r(\gamma)-1}
$$

and the result follows since $r(\gamma) \geq 1$.

As an immediate corollary we have the following result, which is quite similar to a result of Bennequin [Ben].

(1.9) COROLlaRY. If $(b, n)$ is a braid word with exponent sum (or crossing number) equal to $c$, and $e_{\max }$ and $e_{\min }$ are the largest and smallest exponents of $x$ in the Jones polynomial $j(x, y)$ associated to $(b, n)$ then

$$
n-c-1 \geq e_{\max } \geq e_{\min } \geq 1-n-c .
$$

ProOF. Using the equation above for $j(x, y), e_{\max }$ is obtained by choosing 1 for each factor in the expansion of the binomial expression $\left(1+x^{-2}\right)^{r(\gamma)-1}$. This yields the first inequality. Similarly, choosing $x^{-2}$ for each factor yields $e_{\min } \geq$ $n-c-1-2 r+2$ where $r$ is the maximum value of $r(\sigma)$. But $n \geq r$ as we do not increase the number of strands with any allowable move. Thus we have $e_{\min } \geq 1-n-c$, which completes the proof of (1.9).

We also obtain the following estimate on the braid number. Recall that the braid number of a link $l$ is the minimal number $n$ for which there is a braid $(b, n)$ combinatorially equivalent to $l$ (see [Bir]).

(1.10) COROLlaRY. Let $l$ be a link with braid number $\beta$ and Jones polynomial $j(x, y)$. Suppose $e_{\max }$ and $e_{\min }$ are respectively the largest and smallest exponents of $x$ occurring in $j(x, y)$. Then

$$
\beta \geq \frac{\left(e_{\max }-e_{\min }\right)}{2}+1 .
$$

PROOF. If we represent $l$ by a braid $(b, \beta)$ we conclude from (1.9) that

$$
\beta-c-1 \geq e_{\max } \geq e_{\min } \geq 1-\beta-c
$$

where $c$ is the crossing number.

These two inequalities can be written

$$
\beta-c-1 \geq e_{\max }, \quad \beta+c-1 \geq-e_{\min } .
$$

Adding them one gets the inequality of (1.9).

Alternatively, consider the mirror image braid $(\widetilde{b}, \beta)$. Its crossing number is $-c$ and its Jones polynomial $j\left(x^{-1}, y\right)$. Furthermore, $\widetilde{e}_{\min }=-e_{\max }$ and so on. This leads to another proof of (1.9) - except that upon reflection the reader will realize that the two inequalities we have labeled are just interchanged by passing to the mirror image braid.

The following result allows us to read off the number of components of a link from its Jones polynomial. That this is possible is also shown by Theorem 15 of $[\mathbf{J}]$. 
(1.11) PROPOSITION. The smallest exponent of $y$ in $j(x, y)$ is $1-k$ where $k$ is the number of components of the link. Each exponent of $y$ in $j(x, y)$ has the same parity.

ProOF. Choose an invariant computation tree $\Gamma$ whose root is labeled with a braid word corresponding to the given link. The edges are labeled by the monomials $-x^{2},-x^{-2},-x y$, and $-x^{-1} y$. If we set $x=1$ the edges are labeled by -1 and $-y$. There is one (and only one) terminal node $\gamma_{0}$ whose root-path monomial $P\left(\gamma_{0}\right)$ is \pm 1 (when we substitute $x=1$ ). It is the node reached by starting at the root and always choosing the edge labeled -1 . Note that as we descend this path, the number of components in the braids of the nodes we pass never changes, since we are always changing a positive crossing to a negative or vice versa. Thus, $k=r\left(\gamma_{0}\right)$, the number of components of $\gamma_{0}$.

Since we know

$$
j(x, y)=\sum_{\gamma \in T} P(\gamma) \mu^{r\left(\gamma_{0}\right)-1}
$$

where $\mu=-\left(x+x^{-1}\right) / y$ we set $x=1$ and obtain

$$
j(1, y)= \pm(-2 / y)^{r\left(\gamma_{0}\right)-1}+\sum_{\gamma \neq \gamma_{0}} P_{1}(\gamma)(-2 / y)^{r(\gamma)-1}
$$

where $P_{1}(\gamma)$ is $P(\gamma)$ with $x$ set equal to 1 . Thus we have

$$
j(1, y)= \pm(-2)^{k-1} y^{1-k}+\sum P_{1}(\gamma)(-2)^{r(\gamma)-1} y^{1-r(\gamma)}
$$

where each $P_{1}(\gamma) \simeq \pm y^{a}$ for some $a>0$, depending on $\gamma$.

We claim first that the monomial $(-2)^{k-1} y^{1-k}$ does not cancel out with any collection of the monomials in the second term. This is easily seen by reducing all coefficients $\bmod 2^{k}$ since any term in the sum which could cancel would have the form $\pm y^{a}(-2)^{k+a-1} y^{1-k-a}$ and hence would equal $0 \bmod 2^{k}$. Secondly, we assert that none of the monomials

$$
P_{1}(\gamma)(-2)^{r(\gamma)-1} y^{1-r(\gamma)}= \pm(-2)^{r(\gamma)-1} y^{1+a-r(\gamma)}
$$

has $y$ exponent less than $1-k$. This is because as we descend the tree $\Gamma$ from the root to $\gamma$, each time the number of components of braids labeling the nodes increases (by one) we add a factor of $y$ to $P_{1}(\gamma)$. Thus $a \geq r(\gamma)-k$, so $1+a-r(\gamma) \geq 1-k$.

It follows that none of the terms $P(\gamma) \mu^{r(\gamma)-1}, \gamma \neq \gamma_{0}$ has a power of $y$ less than $1-k$ and that $P\left(\gamma_{0}\right)$ has $y^{1-k}$ as a factor and that it does not cancel with any other terms.

To show that all exponents of $y$ have the same parity we return the $x$ 's to the computation tree $\Gamma$.

Now edges of the tree labeled $-x^{2}$ or $-x^{-2}$ correspond to the half of a Conway split which changes the sign of a crossing. This does not change the number of components of the corresponding links. Edges labeled by $-x y$ or $-x^{-1} y$ correspond to removing a crossing which always increases or decreases by 1 the number of components in the corresponding links.

From this it follows that if $\gamma_{0}$ is the root of $\Gamma$, and $\gamma$ is a terminal node with root-path monomial $P(\gamma)= \pm x^{a} y^{b}$ then $k\left(\gamma_{0}\right)$ and $k(\gamma)+b$ have the same parity where $k(\gamma)$ denotes the number of components of the link associated with node $\gamma$. 
The terminal node $\gamma$ is labeled with the empty word on $r(\gamma)$ strands so $k(\gamma)=r(\gamma)$. If $\mu=-\left(x+x^{-1}\right) / y$ then the exponent of $y$ in each term of $P(\gamma) \mu^{r(\gamma)-1}$ is

$$
b-(r(\gamma)-1)=b-(k(\gamma)-1)
$$

which has the same parity as $k\left(\gamma_{0}\right)+1$. Hence the $y$ exponent for each term in $P(\gamma) \mu^{r(\gamma)-1}$ is as claimed. Since

$$
j(x, y)=\sum_{\gamma \in \tau} P(\gamma) \mu^{r(\gamma)-1}
$$

we have the desired result.

Positive braids. Much of our discussion of positive braids relies on the following lemma which we understand has previously been proven by at least two other authors: [vB] and [Ru, p. 34]. As before, we denote the braid on $n$ stands with braid word $b$ by $(b, n)$.

(2.1) LEMMA. Let $(b, n)$ be a positive braid word. By a sequence of invariant Markov moves one can change $(b, n)$ to $\left(b^{\prime}, n^{\prime}\right)$ where $b^{\prime}$ is either the empty word or has the form $b^{\prime}=\alpha \sigma_{i}^{2}$ for some $i$.

PROOF. We define the weight $w$ of a positive braid word by setting $w\left(\sigma_{i}\right)=i$ and $w(\alpha \beta)=w(\alpha)+w(\beta)$. We will prove the lemma by induction on the weight. The only word of weight 0 is the empty word for which the result is true. Suppose it is true for words of weight $<k$ and $b$ has weight $k$.

Let $r$ be the largest integer such that $\sigma_{r}$ occurs in $b$. If $\sigma_{r}$ occurs only once in $b$ then $b$ is conjugate to something of the form $\alpha \sigma_{r}$ where $\alpha$ is positive, and this can be changed to $\alpha$ by an invariant Markov move and $w(\alpha)<k$ so we are done by the inductive hypothesis.

Hence we may assume $\sigma_{r}$ occurs more than once. Let $\sigma_{r} \alpha_{0} \sigma_{r}$ be a subword of $b$ with $\alpha_{0}$ containing no occurrences of $\sigma_{r}$.

There are three cases to consider:

Case 1: $\sigma_{r-1}$ does not occur in $\alpha_{0}$. Then since $\sigma_{r}$ commutes with everything in $\alpha_{0}$ we can replace $\sigma_{r} \alpha_{0} \sigma_{r}$ by $\alpha_{0} \sigma_{r}^{2}$ which is conjugate to a word of the desired form.

Case 2: $\sigma_{r-1}$ occurs only once in $\alpha_{0}$. Then $\sigma_{r} \alpha_{0} \sigma_{r}$ is equivalent to $\alpha_{0}^{\prime} \sigma_{r} \sigma_{r-1} \sigma_{r} \alpha_{0}^{\prime \prime}$ since $\sigma_{r}$ commutes with everything in $\alpha_{0}$ except $\sigma_{r-1}$. This can be replaced by $\alpha_{0}^{\prime} \sigma_{r-1} \sigma_{r} \sigma_{r-1} \alpha_{0}^{\prime \prime}$ which has lower weight. Again we are done by induction.

Case 3: $\sigma_{r-1}$ occurs more than once in $\alpha_{0}$. In this case we choose a subword $\sigma_{r-1} \alpha_{1} \sigma_{r-1}$ of $\alpha_{0}$ with $\alpha_{1}$ containing no occurrences of $\sigma_{r-1}$ (or of $\sigma_{r}$, since it did not occur in $\alpha_{0}$ ). We now restart considering $\sigma_{r-1} \alpha_{1} \sigma_{r-1}$, looking for occurrences of $\sigma_{r-2}$, and checking the three cases. We repeat the process as often as necessary. At each stage we either obtain a word of smaller weight or we decrease the subscript $r$, so the process terminates.

For the study of positive braids, it seems advantageous to codify the information in $j(x, y)$ in a different way which takes advantage of the positiveness. To this end we introduce a polynomial of three variables $R, C, T$ which can be associated to a positive braid and from which various topological invariants are easily read off. This polynomial $J(R, C, T)$ is a true polynomial, i.e., has no negative exponents. Once again we will abuse notation and speak of the polynomial determined by a braid when we mean the polynomial of the associated link. 
(2.2) THEOREM. Associated to a positive braid $(b, n)$ there is a polynomial $J(R, C, T)$ with the following properties:

(1) $J(R, C, T)$ has only positive integer coefficients.

(2) If $j(x, y)$ is the Jones polynomial of $(b, n)$ then

$$
j(x, y)=J\left(-x^{-2},-x^{-1} y,-\left(x+x^{-1}\right) / y\right) .
$$

(3) Conversely, $J(R, C, T)$ depends only on $j(x, y)$, and can be calculated from it.

(4) If $R, C$, and $T$ are assigned degrees 1,2 and -1 respectively, then $J(R, C, T)$ is homogeneous of degree $1-n+c$, where $c$ is the exponent sum of $(b, n)$.

(5) If we set $R=0$, then $J(0, C, T)=C^{p} T^{q}$ where $q$ equals one less than the number of components of the link represented by $(b, n)$.

(6) If $\beta$ is the braid index of $(b, n)$, then $\beta-1 \geq$ the maximum exponent of $T$ in $J(R, C, T)$.

While we are able to prove only the inequality of (6), we would conjecture that for any positive braid its braid number is equal to 1 plus the maximal exponent of $T$ in $J(R, C, T)$. This is equivalent to equality (for positive braids) of the braid number and the number $\left(e_{\max }-e_{\min }\right) / 2$ of Corollary $(1.10)$.

PROOF OF (2.2). In our construction of invariant computation trees (1.7), we first used Conway splits to produce positive braid words and used Lemma (2.1) to apply further Conway splits and finish the construction of the tree. This later process involved only splitting $\alpha \sigma_{i}^{2}$, forming two new nodes labeled $\alpha \sigma_{i}$ and $\alpha$, and forming two new edges to them labeled $-x^{-1} y$ and $-x^{-2}$ respectively. Hence, if we start with a positive braid $(b, n)$, we can construct an invariant computation tree $\Gamma$ with root labeled by $(b, n)$ and the additional property that if $\gamma$ is a nonterminal node, the two edges emanating from it are labeled $-x^{-2}$ and $-x^{-1} y$.

We now alter the labelings, replacing each $-x^{-2}$ with $C$ and each $-x^{-1} y$ with $R$. We can then define a polynomial $J(R, C, T)$ (which at this point may seem to depend on the choice of computation tree) by the following formula:

$$
J(R, C, T)=\sum_{\gamma \in \tau} P(\gamma) T^{r(\gamma)-1}
$$

In this formula, $\tau$ is the set of terminal nodes of $\Gamma, r(\gamma)$ is the number of components of the trivial link with which $\gamma$ is labeled and $P(\gamma)$ is the root-path monomial of $\gamma$, i.e., $P(\Gamma)=R^{p} C^{q}$ for some $p, q \geq 0$.

Property (1) of the theorem is immediate and property (2) is also obvious, given our way of calculating $j(x, y)$ from a computation tree.

The proof of (4), that $J(R, C, T)$ is homogeneous, is essentially identical to the proof of (1.5) and (1.6). We note that if an edge going from a vertex labeled $(b, n)$ to one labeled $\left(b^{\prime}, n^{\prime}\right)$ has $C$ as its label, then since $\chi=n-c$ (from (1.4))

$$
\chi(b, n)=\chi\left(b^{\prime}, n^{\prime}\right)-2=\chi\left(b^{\prime}, n^{\prime}\right)-\operatorname{deg} C .
$$

Similarly, if the edge is labeled $R$

$$
\chi(b, n)=\chi\left(b^{\prime}, n^{\prime}\right)-1=\chi\left(b^{\prime}, n^{\prime}\right)-\operatorname{deg} R .
$$

Inductively, if the root $\gamma_{0}$ is labeled by $(b, n)$ and $\gamma$ is any node with root-path monomial $P(\gamma)$, then

$$
\chi(b, n)=\chi\left(b^{\prime}, n^{\prime}\right)-\operatorname{deg} P(\gamma)
$$


so

$$
\operatorname{deg} P(\gamma)=-\chi(b, n)+\chi\left(b^{\prime}, n^{\prime}\right) .
$$

If $\gamma$ is a terminal node with $r(\gamma)$ components, then $\chi\left(b^{\prime}, r(\gamma)\right)=r(\gamma)$. Hence

$$
-\chi(b, n)=\operatorname{deg} P(\gamma)-r(\gamma)=\operatorname{deg}\left(P(\gamma) T^{r(\gamma)-1}\right)-1 .
$$

Since

$$
J(R, C, T)=\sum_{\gamma \in \tau} P(\gamma) T^{r(\gamma)-1},
$$

it follows that $J(R, C, T)$ is homogeneous of degree $1-\chi(b, n)$. Now, $\chi(b, n)=n-c$ where $c$ is the exponent sum of the word $b$. If $b$ determines a knot, then this is well known (see [B-W]) to equal $-2 g$, where $g$ is the genus of $(b, n)$. It follows that $J$ is homogeneous of degree $2 g$.

We can now prove property (3), which will prove in addition that $J(R, C, T)$ is independent of the choice of invariant computation tree. By Theorem (1.8) we know that there are unique numbers $a_{i j}$ such that

$$
j(x, y)=x^{\alpha} \sum a_{i j} y^{i}\left(1+x^{-2}\right)^{j}
$$

where $\alpha=n-c-1=-2 g$. Hence

$$
\begin{aligned}
j(x, y) & =\sum a_{i j} x^{\alpha-j} y^{i+j}\left(\left(x+x^{-1}\right) / y\right)^{j} \\
& =\sum(-1)^{i} a_{i j} x^{\alpha+i}(-y / x)^{i+j}\left(-\left(x+x^{-1}\right) / y\right)^{j} \\
& =\sum(-1)^{\frac{1-\alpha}{2}} a_{i j}\left(-x^{-2}\right)^{-\frac{i+\alpha}{2}}(-y / x)^{i+j}\left(-\left(x+x^{-1}\right) / y\right)^{j} .
\end{aligned}
$$

Substituting $R=-y / x, C=-x^{-2}$ and $T=-\left(x+x^{-1}\right) / y$ we get

$$
j(x, y)=\widetilde{J}(R, C, T)=\sum(-1)^{\frac{i-\alpha}{2}} a_{i j} R^{i+j} C^{-\frac{i+\alpha}{2}} T^{j} .
$$

Thus $\widetilde{J}$ is homogeneous of degree $-\alpha$ if $C, R$, and $T$ are given the weights 2, 1 , and -1 respectively. Since the $\left\{a_{i j}\right\}$ are unique, $\widetilde{J}$ is the unique polynomial (in $R, C^{1 / 2}$, and $T$ ) with this homogeneity property and the property that $\widetilde{J}\left(-x^{-1} y,-x^{-2},-\left(x+x^{-1}\right) / y\right)=j(x, y)$. We have shown that $J(R, C, T)$ also has these properties, so it follows that $J=\widetilde{J}$. Notice one can conclude that $i-\alpha$ is even and $(-1)^{\frac{i-\alpha}{2}} a_{i j} \geq 0$. These are not difficult to show directly, however. Showing property (5) is like the proof of (1.11), only easier. We use the fact that

$$
J(R, C, T)=\sum_{\gamma \in \tau} P(\gamma) T^{r(\gamma)-1}
$$

There is precisely one $\widehat{\gamma} \in \tau$ for which $P(\gamma)$ contains no factor of $R$, namely $\widehat{\gamma}$ is the terminal node reached by starting at the root of $\Gamma$ and moving down the tree, always choosing the edge labeled $C$. Clearly for every other $\gamma \in \tau, P(\gamma)$ will be of the form $R^{a} C^{b}, a>0$, and $P(\widehat{\gamma})=C^{p}$ for some $p$. The edges of $\Gamma$ labeled by $C$ correspond to the part of the Conway split which replaces a left-handed crossing with a right-handed one.

This does not change the number of components in the corresponding link. Thus the link corresponding to the root and the link corresponding to $\widehat{\gamma}$ have the same number of components. 
By the formula for $J$ above we conclude

$$
J(0, C, T)=C^{p} T^{r(\widehat{\gamma})-1} .
$$

Since $\widehat{\gamma}$ is a terminal node the link associated with it has $r(\widehat{\gamma})$ components which is the same as the number of components $q$ of the link associated to $(b, n)$.

Finally, to prove (6) one could mimic the proof of (1.8) and (1.10). Alternatively, we observe that from property (2) it follows that if $i=\sqrt{-1}$,

$$
j(i x,-i y)=J\left(x^{-2}, x^{-1} y,\left(x^{-1}-x\right) / y\right) .
$$

Since $J$ has positive coefficients, it follows that twice the largest power of $T$ in $J(R, C, T)$ equals the difference of the largest and smallest power of $x$ in $j(i x,-i y)$. Since this is the same as $e_{\max }-e_{\min }$ for $j(x, y)$ as defined in (1.10), the result follows.

We will refer to the computation tree constructed at the beginning of $(2.2)$ as a positive computation tree. In particular, it has every node labeled with a positive braid word and every edge is labeled with $R$ or $C$.

(2.3) LEMMA. Suppose $\Gamma$ is a positive computation tree whose root is labeled with $(b, n)$. If any node of $\Gamma$ is labeled with a braid word whose associated link has $n$ components, then $n$ is the braid number of $(b, n)$.

Proof. Suppose $\gamma$ is the node associated with a positive braid-word $\left(b_{0}, n_{0}\right)$ whose link has $n$ components. Then if $J_{0}$ is the polynomial of (2.2) corresponding to $\left(b_{0}, n_{0}\right)$, we know that there is a term of $J_{0}$ with $T$ exponent equal to $n-1$ (by (5) of (2.2)). But if $J(R, C, T)$ is the polynomial associated with $(b, n)$ then using $\Gamma$ to compute $J(R, C, T)$ we get $J(R, C, T)=P_{1} J_{0}+P_{2}$ where $P_{1}, P_{2}$ are positive polynomials in $R, C, T$. It follows that the largest exponent of $T$ in $J(R, C, T)$ is at least $n-1$.

Thus, if $\beta$ is the braid number of $(b, n)$, we have by (6) of $(2.2)$

$$
\beta \geq n \text {. }
$$

On the other hand, $(b, n)$ is represented with $n$ strands, so $\beta \leq n$.

We now investigate positive braids $(b, n)$, where $b$ contains a full twist, $\Delta^{2}$ (see [Bir] for a definition).

(2.4) COROLLARY. Let $(b, n)$ be a positive braid on $n$ strands with $b=a \Delta^{2}$, where $\Delta^{2}$ is the "full twist" word. Then $n$ is the braid number of $(b, n)$.

PROOF. By (2.3) it suffices to show there is a positive computation tree $\Gamma$ which contains a node labeled by $\left(\Delta^{2}, n\right)$, since the link associated with $\left(\Delta^{2}, n\right)$ has $n$ components.

The word $\Delta^{2}$ commutes with every element of the braid group $B_{n}$ and given $i<n, \Delta^{2}$ is equivalent in the braid group to a positive word $D(i)$ which begins with the generator $\sigma_{i}$ (see (2.4.1) of [Bir]).

We write $b$ as $a \Delta^{2}$ and prove the existence of the desired positive computation tree by induction on the length of $a$. If $a$ has length 0 , the result is trivial.

Otherwise, $a=a^{\prime} \sigma_{i}$ for some $i$, so

$$
a \Delta^{2}=a^{\prime} \sigma_{i} \Delta^{2}=a^{\prime} \sigma_{i} D(i)=a^{\prime} \sigma_{i}^{2} D^{\prime}(i)
$$


where $D^{\prime}(i)$ is given by $D(i)=\sigma_{i} D^{\prime}(i)$ and = means in the semigroup of positive braids on $n$ strands. If we apply a Conway split to this word, one of the child nodes is labeled $a^{\prime} \sigma_{i} D^{\prime}(i)=a^{\prime} D(i)=a^{\prime} \Delta^{2}$. Since the length of $a^{\prime}$ is less than the length of $a$, we can by the induction hypothesis complete this branch of the tree in such a way that one node will be labeled with $\Delta^{2}$.

REMARK. Corollary 2.4 had been conjectured for some time. Some time ago, Hugh Morton (private communication) showed that $n$ shows up as a gap in the Alexander polynomial and thus is a knot invariant.

\section{BIBLIOGRAPHY}

[Ben] D. Bennequin, Entrelacement et structures de contact, Thesis, Paris, 1982.

[Bir] J. Birman, Braids, links and mapping class groups, Ann. of Math. Studies, no. 82, Princeton Univ. Press, Princeton, N. J., 1984.

[B-W] J. Birman and R. Williams, Knotted periodic orbits I: Lorenz knots, Topology 22 (1983).

[FYHLMO] P. Freyd et al., A new polynomial invariant of knots and links, Bull. Amer. Math. Soc. 12 (1985), 239-246.

[J] Vaughan Jones, A polynomial invariant for knots via von Neumann algebras, Bull. Amer. Math. Soc. 12 (1985), 103-111.

[L-M] W. B. R. Lickorish and K. Millett, A polynomial invariant of oriented links, MSRI preprint \#04212-85, February 1985.

[M] H. R. Morton, Closed braid representatives for a link and its Jones-Conway polynomial, preprint, 1985.

[R] D. Rolfsen,Knots and links, Publish or Perish, Berkeley, Calif., 1976.

[Ru] L. Rudolph; Braided surfaces and Seifert Ribbons for closed braids, Comm. Math. Helv. 1983.

[vB] J. M. van Buskirk, private communication.

Department of Mathematics, NorthWestern University, EVANSton, Illinois 60201 